INNOVATION AND IMPROVEMENT

\title{
Improvement Happens: An Interview with Deeb Salem, MD and Brian Cohen, MD
}

\author{
Matthew J. Press, MD, MSc \\ Departments of Public Health and Medicine, Weill Cornell Medical College, New York, NY, USA.
}

$\mathrm{J}$ Gen Intern Med 27(3):381-5

DOI: $10.1007 / \mathrm{s} 11606-011-1947-7$

(C) Society of General Internal Medicine 2011

$T^{n}$ he idea of concierge medicine, where patients pay a surcharge for increased access and additional services from their physician, elicits a range of reactions in the medical community. Ethical concerns about patient abandonment and the creation of a two-tiered health care system have been raised, but the appeal of more time and support for personalized attention is a draw for some patients and physicians. In addition, some say that concierge medicine raises the bar for primary care practice writ large by reminding us of what patients want and what primary care practice can be.

At the Tufts Medical Center in Boston, a new model of concierge medicine seeks to resolve the differences in opinion. Opened in 2004, the Tufts academic retainer practice is by all appearances a typical primary care concierge practice. The difference is that it operates along with a traditional academic general medicine practice, and its revenues go towards the delivery of care in both settings. This cross-subsidization, according to the practice's leaders, has helped expand access to care in the traditional practice, which serves an economically diverse population. Currently, 500 patients pay the $\$ 2,000$ annual fee to be in the retainer practice, while 35,000 patients are in the traditional practice.

In this installment of Improvement Happens, a periodic feature of JGIM sponsored by the California Healthcare Foundation, JGIM spoke with Deeb Salem, MD (Fig. 1), and Brian Cohen, MD (Fig. 2), about the services, ethics, and implications of the Tufts academic retainer practice. Dr. Salem is Chairman of the Department of Medicine at Tufts University School of Medicine and the co-founder of the Tufts academic retainer practice, and Dr. Cohen is medical director for the practice.

Received September 7, 2011

Revised October 27, 2011

Accepted October 31, 2011

Published online December 9, 2011

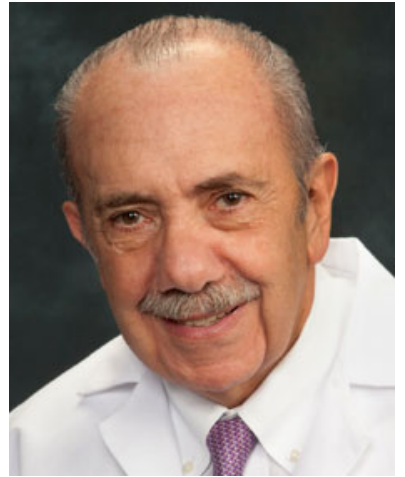

Figure 1. Dr. Deeb Salem.

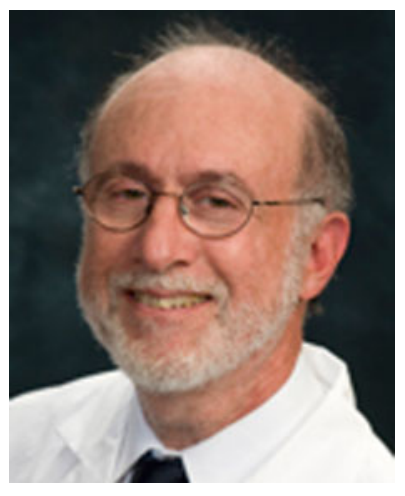

Figure 2. Dr. Brian Cohen.

JGIM: How did the Tufts academic retainer practice begin?

Deeb Salem: In 2003, we saw the first of some concierge practices developed in the Boston area by a company called MDVIP. At first I said, "There is no place for this in an academic medical center with multiple missions." But after thinking about it, the concept of a "mission-driven" concierge practice came to my mind. So I called the CEO of MDVIP and said, "We're interested, but only under some new rules."

JGIM: What do you mean by "mission-driven" concierge medicine?

DS: Ours is the third oldest hospital in the country and was founded with the idea that people of means supported the care of people that had no means. The original idea for our concierge practice was in line with this mission. If we 
could make the concierge practice financially successful, then the revenue would be used to support what we all know is an underfunded part of American healthcare: primary care. This isn't unlike the way a cath lab can be a "cash cow" for divisions of cardiology.

JGIM: How is the Tufts academic retainer practice different than the typical concierge medical practice?

DS: The abandonment of patients in typical concierge practice bothered me. When a practice switches to concierge, the provider usually notifies his or her patients that if they don't sign up and pay the fee, then they'll have to find a new primary care physician (PCP). We said, "Why does that have to happen?" So we took three of our PCPs and had them split their time between the concierge practice and our traditional practice. We sent their patients a letter offering them the option of being seen in a different practice setting, with better ambience and other extra features, or staying in the regular practice.

JGIM: Describe the experience for the typical patient in the concierge practice.

Brian Cohen: We try to get patients in soon after they sign up for a one-hour initial visit, which includes a physical, EKG, bloodwork, reviewing their records, and getting to know them. Then, we put together a comprehensive wellness and prevention plan for them. There's so much we can do by phone after the initial visit.

JGIM: How is that different than a patient's experience in the traditional general medicine practice?

BC: In the traditional practice, there's less time for each office visit, and, while patients with an urgent medical issue will be seen quickly, they may not be seen by their own PCP (Fig. 3). In the concierge practice, the patient sees their own PCP $95 \%$ of the time - and patients can be seen same day or next day, regardless of whether it's medically urgent (Fig. 4). We also have a lot more time to arrange specialist visits and coordinate care. Although most patients in the concierge practice don't have our cell phone numbers, they

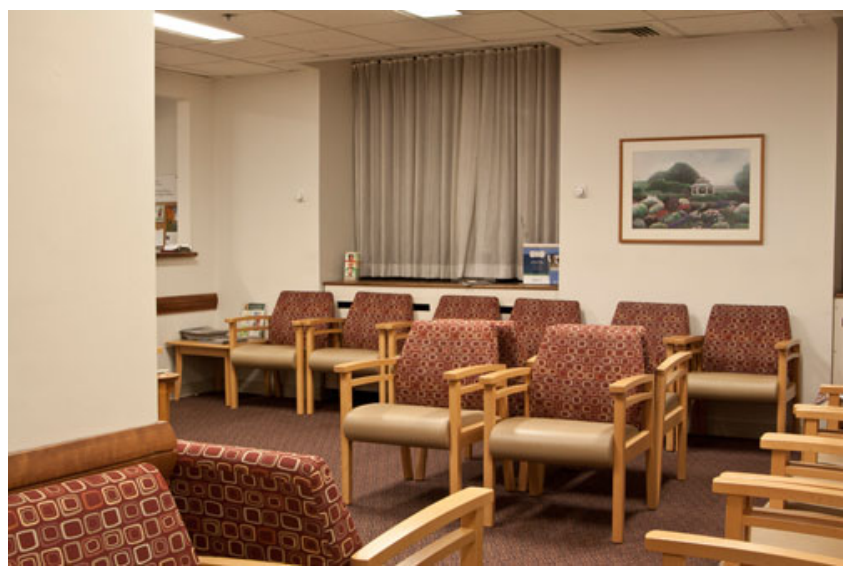

Figure 3. Traditional Practice Waiting Room.

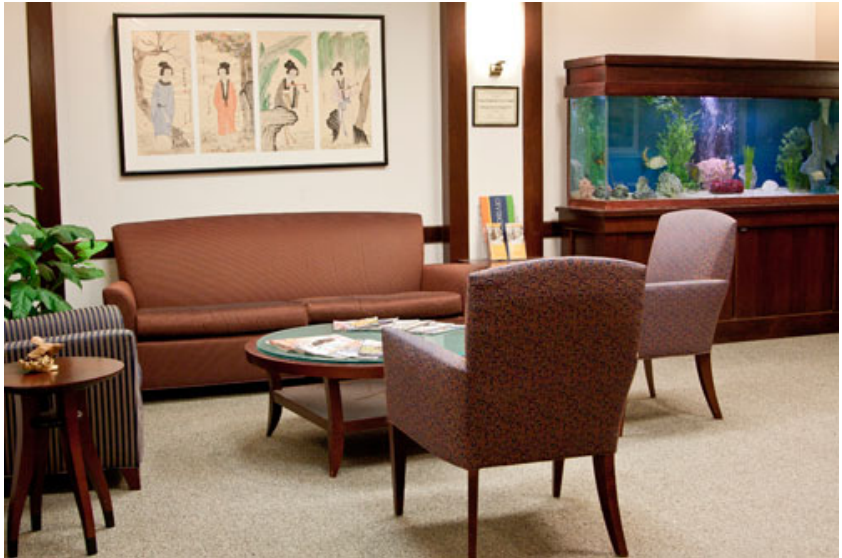

Figure 4. Concierge Practice Waiting Room.

can be put through to their own doctor by the covering doctor when they call. So, the level of service and the level of access are much different in the concierge practice than in the traditional practice.

DS: Patient satisfaction is higher in the concierge practice in part because we are able to more immediately respond to phone calls and spend more face-to-face time with patients. Patients in the traditional practice also have 24-hour access to a covering physician, just not necessarily to their own physician.

JGIM: This sounds a bit like a two-tiered system, which arguably goes against the mission you discussed earlier.

DS: That's a common criticism, but tiering is already all over the place in health care. That's nothing new. We believe that our system has raised the level of quality for everybody. For example, we've been able to improve access to care in the traditional practice by using some revenue from the concierge practice to contribute towards the hiring of additional PCPs. In fact, some concierge patients have chosen our practice in particular because they know their money is going to help others, and some have even made extra donations specifically towards primary care. That helps advance our mission.

BC: No one is getting scarce medical resources ahead of anybody else. The difference between the two practices is more about convenience and time.

DS: In the concierge practice, the physician is able to pick up the phone and arrange a test or appointment, which typically makes the response much more rapid.

JGIM: Shouldn't all patients be entitled to that level of service?

BC: Absolutely. But, I don't think it's feasible given the economics of primary care. We can do that in the concierge practice because we have the additional personnel that are necessary. The concierge practice is a niche, and I don't think it's reducing access or quality of care for other people.

DS: In many academic medical centers, high-profile patients get special service, but they don't call it concierge. 
These patients might donate money to the hospital, but not to the primary care group. In our system, we have an official mechanism in place to fund that level of service.

JGIM: Were any of the physicians at Tufts upset when you started the concierge practice?

BC: There were some concerns initially. But the fact that we set this practice up to help subsidize the traditional practice helped ease those concerns. It took a little while to get people to understand why we were doing this. But, for the most part, everybody is on-board with it.

DS: Plus, the amount of patients we see in the concierge practice is such a small percentage of the patients we see overall. But, some of the physicians we asked in the beginning if they were interested in participating said they were not.

JGIM: Was there pushback from any patients?

BC: There was from a couple of patients, who were upset that I was splitting my time. They sort of felt like second class citizens. But most patients were very appreciative of the fact that we split our time and were still able to see them in the traditional practice.

DS: In other concierge practices, patients are told they're in or they're out. Here, patients were able to continue seeing their own doctor either way.

JGIM: But didn't you have to dismiss some patients in order to clear half of your schedule for the concierge practice?

BC: No. I didn't reduce my panel size when we started. I reduced the time I spent in the traditional practice, which meant that my patients were seen more often by other physicians, especially for urgent care visits. But I worked more efficiently to see more of them while I was in the traditional practice. I also stopped taking new patients in the traditional practice.

DS: Being in the concierge practice also gave these physicians more time to take care of their traditional patients. So, even the patients who did not pay to be in the concierge practice benefitted by getting a little more attention than they would have had before. If the concierge practice were to expand rapidly in the future, then some patients in the traditional practice might have to be transferred to other physicians. But we have the capacity for that, in part because we've been able to hire more physicians since starting the concierge practice.

JGIM: Are you worried that patients in the concierge practice are getting too much care and driving up health care costs?

DS: This was a concern for the insurance companies when we first started. But, while I don't have the data to say for sure, I think it has been quite the opposite. Say a patient comes in with unusual chest pain symptoms, and the physician would need to spend half an hour explaining that it is not cardiac. In a busy clinic setting, you might not have the time to do that and might send them for a stress test instead. But, in the concierge practice, you do have the time. The lack of time is one of the big problems in outpatient internal medicine, and it's because of the flawed financial model. If internists could afford to spend more time with patients, I truly believe costs would come down. It's the way primary care should be.

BC: We don't recommend anything that we wouldn't be recommending to anyone else in the same medical circumstances. We look at their overall picture, recommend appropriate screening tests, and then spend a little more time talking about nutrition and lifestyle. We have more time to go into that kind of detail. But, there are some lab tests we do for concierge patients that are not covered by insurance as part of a routine physical.

JGIM: Which tests are these, and why do you do them?

BC: Tests like TSH, calcium, and liver function tests will generally not be covered by insurance unless there are specific symptoms that would dictate their being ordered. We believe these are worthwhile tests. Doing these tests has virtually no downstream complications and is paid for by the patient's annual fee.

JGIM: How does the work day in the concierge practice compare to the traditional practice?

BC: In the traditional practice, I typically saw ten to twelve patients in a half day session. I had two sessions a day, three or four days a week. In the concierge practice, I see three or four patients in a half day session, probably four or five times a week, with the rest of the time available for administrative work. Some of that administrative time is for my responsibilities as director of the practice, but much of it is spent following up on patient visits. I spend time making sure that specialists have all the information they need to evaluate patients I refer to them. I also follow up on specialty visits and help patients make decisions based on specialists' recommendations. When my patients are admitted, a hospitalist is the attending of record, but I stay involved with their care, giving the ward team input from someone who knows the patient well. They appreciate that, and it helps with discharge planning and the transition from inpatient to outpatient.

JGIM: Has career satisfaction improved for the physicians who now spend at least half of their time in the concierge practice?

BC: One of the reasons physicians go into concierge medicine is to get off the treadmill — not being able to keep up with seeing ten to twelve patients a session, not feeling like you can do an in-depth, good job. Having that extra time has made the experience very satisfying. But, doing the split model between concierge and traditional is, in some ways, quite difficult. For instance, you might be in the concierge practice and get an urgent message from the traditional practice, where you don't have as much secretarial help. The close proximity of the two practices 
and the shared electronic medical record helps, but it's still not easy. We have not had the leisurely concierge life one might picture.

JGIM: How does the concierge practice fit in with the teaching mission of an academic medical center?

DS: Our amount of teaching has increased because we have more physicians and are able to give those who like to teach more time for that.

JGIM: Do you worry about the message having a concierge practice sends to medical students?

BC: The students are for the most part unaware of its presence because we're located in a separate area from the traditional practice. But, again, the way we've set this up as a practice is to try to subsidize the traditional practice. I think that that helps address criticism from the equity point of view.

DS: Equity sometimes collides with being able to do more for more patients. I think the care for all of our primary care patients is better because of the added money from the concierge practice. The well-off might be a little better off, but everyone else is better off too. I wouldn't be doing it if that wasn't the case.

JGIM: What were the start-up costs involved with the original launch of the concierge practice?

DS: The board of trustees gave us $\$ 300,000$ to build the facility. A number of members of the board are now patients in the concierge practice. As a result, they have gained a stronger connection to primary care and have been more willing to support it in general than ever before.

JGIM: Could a practice without access to that kind of start-up money pull off opening a hybrid conciergetraditional model like you did?

DS: You do have to have some investment to upgrade the facility and hire staff. The money may not come from donors, like it did for us; it could be the practice itself investing, if they think they have a willing patient population. We serve a mixed population: from the homeless to business people and baby-boomers moving into the city from the suburbs. So we were fortunate to have enough patients who could afford to enter the concierge practice.

BC: Private practices thinking about becoming concierge practices often do surveys to see what percentage of their panel will be willing to switch. They keep their same offices and fill the practice mostly with patients from their old practice. For us, only a very small percentage of patients transferred over early on. We mostly had to recruit patients from outside the traditional practice. We could have lost money because of this; however, one of the advantages of doing the split model was that the concierge practice only had to cover half of the physicians' salaries. The traditional practice covered the other half. That really helped the finances during the first couple of years.

JGIM: Can it be done without the help of a concierge company?

DS: It can be done without an outside company but does require help from legal and business experts. When we started, nobody that we knew of had ever done it this way before. But we have proven it can be done. It would be much easier to do now than when we started.

BC: The company was very helpful at the beginning with marketing, although, in retrospect, we didn't really get many patients from cable TV ads and print ads. We've been more successful using word of mouth and local connections.

DS: We would be glad to host visitors from other institutions to see how our system works.

JGIM: Is the concierge practice consistent with a patientcentered medical home?

DS: I'm very supportive of the medical home, but I'm concerned about who will pay for it. One advantage of the concierge model is that it provides funding for medical home infrastructure. The physicians there have the time to navigate patients through the health care system.

JGIM: If this hybrid model spread across the country, could it worsen the primary care shortage?

DS: Actually, we see 5,000 more patients now than we did before we started the concierge practice in part because we have been able to hire several new PCPs. Plus, comparatively few patients are willing to pay the concierge fee, so concierge medicine is still a niche-at least for now. If funding for primary care decreases, and a lot of patients are dissatisfied with their care, concierge medicine might grow - and the primary care shortage might worsen. But we're hoping that is not what will happen to primary care. Hopefully, the opposite will occur, and primary care physicians will become better compensated - which would lead more physicians to choose careers as PCPs. In fact, we have been able to increase the salaries of all of our PCPs.

JGIM: What have been some challenges to the success of this model?

DS: Recruiting patients. We had an initial bolus of patients enter the concierge practice, and it has continued to grow. But I wish the slope of growth was a little steeper.

BC: One issue is getting the right staffing to provide a higher level of service. That can be costly, and-in an academic medical center-negotiating human resources can be a little tricky. Another challenge is explaining to patients in the concierge practice that while they have same- or nextday access to primary care, we cannot promise the same access to specialists. But, you know what? That is what they want. The wait to see a specialist (and for testing, such as 
MRIs or stress tests) is longer than patients would like, especially once we've spoiled them with the access to primary care.

JGIM: How has being involved with this new model changed your view of primary care?

BC: It has let me do primary care the way I want to do it. I think of myself more as a general internist than a primary care physician. I love doing preventive medicine and physicals, but I also love coordinating the care of people who see multiple specialists. That is what I can do well in this practice.

Editor's note: Interview responses have been edited.

Corresponding Author: Matthew J. Press, MD, MSc; Departments of Public Health and Medicine, Weill Cornell Medical College, 402 E. 67th St., New York, NY 10065, USA (e-mail: Map9069@med.cornell.edu). 